\title{
The structure of protoplanetary disks surrounding three young intermediate mass stars ${ }^{\star}$
}

\section{Resolving the disk rotation in the [OI] $6300 \AA$ line}

\author{
G. van der Plas ${ }^{1,2}$, M. E. van den Ancker ${ }^{1}$, D. Fedele ${ }^{1,3,4}$, B. Acke ${ }^{5}$, \\ C. Dominik ${ }^{2}$, L. B. F. M. Waters ${ }^{2}$, and J. Bouwman ${ }^{4}$ \\ 2 Sterrenkundig Instituut "Anton Pannekoek", University of Amsterdam, Kruislaan 403, 1098 SJ Amsterdam, The Netherlands \\ Dipartimento di Astronomia, Universitá Degli Studi Di Padova, Vicolo Dell` Osservatorio 2, 35122 Padova, Italy
}

1 European Southern Observatory, Karl-Schwarzschild-Str.2, 85748 Garching bei München, Germany e-mail: gvanderp@eso.org

4 Max-Planck-Institut für Astronomie, Königstuhl 17, 69117 Heidelberg, Germany

5 Institute of Astronomy, KU Leuven, Celestijnenlaan 200D, 3001 Leuven, Belgium ${ }^{\star \star}$

Received 18 October 2007 / Accepted 4 February 2008

\section{ABSTRACT}

\begin{abstract}
We present high-spectral-resolution, optical spectra of three young, intermediate-mass stars, in all of which we spectrally resolve the $6300 \AA[\mathrm{OI}]$ emission line. Two of these have a double-peaked line-profile. We attempt to fit these data using a simple model of $[\mathrm{OI}]$ emission, which is generated by photo-dissociation of $\mathrm{OH}$ molecules, in the upper layer of a circumstellar disk, by stellar UV radiation. We translate the Doppler-broadened [OI] emission line profile, into a measure of emission, as a function of distance from the central star. The resulting spectra are in agreement with the expected disk shapes derived from their spectral energy distribution. We find evidence for shadowing by an inner rim in the disk surrounding HD 101412, and observe a flaring disk structure in HD 179218, while the $[\mathrm{OI}]$ spectrum of HD $135344 \mathrm{~B}$ is more complex. The [OI] emission starts for all three targets at velocities corresponding to their dust sublimation radius and extends up to radii of 10-90 AU. This shows that this method can be a valuable tool in the future investigation of circumstellar disks.
\end{abstract}

Key words. stars: circumstellar matter - stars: pre-main sequence - stars: planetary systems: protoplanetary disks stars: planetary systems: formation - stars: abundances - stars: general

\section{Introduction}

HD 101412, HD 135344 B and HD 179218 are three young and isolated, intermediate-mass stars. Two of these stars, HD 101412 and HD 179218 are Herbig Ae/Be (HAEBE) stars, while the third star HD 135344 B (often incorrectly referred to as HD 135344 in the literature), is a classical T Tauri star of spectral type F. HAEBE stars are the more massive counterparts of T Tauri pre-main-sequence stars with typical masses of 2-8 $M_{\odot}$. Their spectral energy distribution (SED) is characterized by the presence of an infrared excess due to thermal re-emission of circumstellar dust, which is thought to be the signature of a circumstellar disk. Previous studies of these stars and their circumstellar disks have focused mainly on this dust (e.g. Hillenbrand et al. 1992; Natta et al. 2001; Mannings \& Sargent 1997; Meeus et al. 2001).

Dust, however, accounts for only $1 \%$ of the mass of the disk, and it is unclear whether the dust is coupled to the remaining $99 \%$ of the disk mass, the gas. To be able to analyze the coupling between dust and gas and improve our understanding of circumstellar disks, it is necessary to study the dust and gas in circumstellar disks simultaniously. The study of the physics

\footnotetext{
* Based on observations collected at the European Southern Observatory, Paranal, Chile. (Program ID 077.C-0521A).

$\star \star$ Postdoctoral Fellow of the Fund for Scientific Research, Flanders.
}

and chemistry of disks furthermore provides a measure of their chemical abundances and dynamics. Traditionally, it has been difficult to study circumstellar gas, but the development of largeaperture mirrors and more efficient spectrographs has enabled several gas diagnostics to be accurately determined. These include hot and cold CO gas (e.g. Bik \& Thi 2004; Blum et al. 2004; Blake \& Boogert 2004; and Najita et al. 2003), hot water (e.g. Carr et al. 2004), and molecular hydrogen (e.g. Carmona et al. 2008; Bitner et al. 2007; Martin-Zaïdi et al. 2007).

Acke et al. (2005), and Acke \& van den Ancker (2006), have added molecular oxygen to this list. They have developed a method for examining excited, neutral, oxygen atoms in the surface of a flared, rotating passive disk, and have tested the method two HAEBE stars: HD 97048 and HD 100546. Applying their method, they resolve the disk rotation, and the distribution of emitting gas, in the surface layer of the circumstellar disk, and find evidence for a gap in the disk surrounding HD 100546, which they interpret as due to the presence of an orbiting body of planetary mass, within the circumstellar disk.

In this paper, we apply the method of Acke et al. (2005) to three more stars, one of which has a non-flaring (flat) disk geometry. We first describe the stars, our observations and data reduction in Sects. 2 and 3, then proceed to describe the method and the resulting spectra in Sect. 4 , and discuss our results in Sect. 5. We observed our targets using VLT/MIDI, to obtain 
Table 1. Log of spectroscopic observations with UVES.

\begin{tabular}{ccccccc}
\hline \hline Object & $\begin{array}{c}\text { Date } \\
\text { dd/mm/yy }\end{array}$ & $\begin{array}{c}\text { UT } \\
\text { hh:mm:ss }\end{array}$ & $\begin{array}{c}\text { Exp. time } \\
{[\mathrm{s}]}\end{array}$ & PA & B & $S / N$ \\
& $\left.{ }^{\circ}\right]$ & & \\
\hline HD 101412 & $15 / 05 / 06$ & $23: 37: 48$ & 300 & 0 & B1 & 174 \\
& $15 / 05 / 06$ & $23: 43: 45$ & 300 & 0 & B1 & 199 \\
& $15 / 05 / 06$ & $23: 50: 04$ & 300 & 0 & B1 & 189 \\
& $15 / 05 / 06$ & $23: 56: 01$ & 300 & 0 & B1 & 175 \\
& $17 / 05 / 06$ & $00: 39: 17$ & 300 & 90 & B1 & 183 \\
& $17 / 05 / 06$ & $00: 45: 15$ & 300 & 90 & B1 & 207 \\
& $17 / 05 / 06$ & $00: 51: 13$ & 300 & 90 & B1 & 155 \\
& $17 / 05 / 06$ & $00: 57: 09$ & 300 & 90 & B1 & 199 \\
& $03 / 07 / 06$ & $23: 52: 01$ & 300 & 0 & B2 & 149 \\
& $03 / 07 / 06$ & $23: 58: 00$ & 300 & 0 & B2 & 141 \\
& $04 / 07 / 06$ & $00: 03: 58$ & 300 & 0 & B2 & 161 \\
& $04 / 07 / 06$ & $00: 09: 53$ & 300 & 0 & B2 & 151 \\
& $04 / 07 / 06$ & $00: 18: 58$ & 300 & 90 & B2 & 137 \\
& $04 / 07 / 06$ & $00: 24: 53$ & 300 & 90 & B2 & 144 \\
& $04 / 07 / 06$ & $00: 30: 48$ & 300 & 90 & B2 & 135 \\
& $04 / 07 / 06$ & $00: 36: 46$ & 300 & 90 & B2 & 155 \\
\hline HD 135344 B & $17 / 05 / 06$ & $04: 19: 43$ & 300 & 0 & B1 & 197 \\
& $17 / 05 / 06$ & $04: 25: 39$ & 300 & 0 & B1 & 196 \\
& $17 / 05 / 06$ & $04: 35: 08$ & 300 & 90 & B1 & 234 \\
& $17 / 05 / 06$ & $04: 41: 04$ & 300 & 90 & B1 & 262 \\
& $16 / 06 / 06$ & $03: 49: 40$ & 300 & 0 & B2 & 144 \\
& $16 / 06 / 06$ & $03: 55: 36$ & 300 & 0 & B2 & 143 \\
& $16 / 06 / 06$ & $04: 06: 12$ & 300 & 90 & B2 & 227 \\
& $16 / 06 / 06$ & $04: 12: 29$ & 300 & 90 & B2 & 240 \\
\hline HD 179218 & $17 / 05 / 06$ & $06: 50: 00$ & 180 & 0 & B1 & 309 \\
& $17 / 05 / 06$ & $06: 56: 28$ & 180 & 90 & B1 & 237 \\
& $16 / 06 / 06$ & $04: 22: 43$ & 180 & 0 & B2 & 293 \\
& $16 / 06 / 06$ & $04: 28: 05$ & 180 & 90 & B2 & 251 \\
\hline
\end{tabular}

spatially-resolved observations of the $10 \mu \mathrm{m}$ silicate bump, which corresponds to emission from warm dust in the same region as the [OI] emission. The results of these interferometric observations will be presented in Fedele et al. (2008, in prep.; Paper II).

\section{Observations and data reduction}

We obtained high-spectral-resolution échelle spectra of HD 101412 , HD 135344 B and HD 179218 during 4 nights, using the KUEYEN $8.2 \mathrm{~m}$ ESO VLT and UVES spectrograph (Dekker et al. 2000). The observation log is presented in Table 1. The data was reduced using the $\mathrm{UVES}^{1}$ pipeline ${ }^{2}$, which performs wavelength calibration, order extraction, background subtraction and flatfield correction. We completed four observations of each targeted star. Half of these observations were acquired with the spectroscopic slit unrotated (at 0 degrees), and the remainder with the slit rotated by 90 degrees. The spectra have a spectral resolution of $\frac{\lambda}{\Delta \lambda}=77000$, determined using the telluric absorption lines about the $6300 \AA$ [OI] feature. All three sources are spatially-unresolved. We derive upper limits on their angular size, using a $3 \sigma$ detection limit, and following the same procedure as e.g. Hirth et al. (1994), Hirth et al. (1997) and Acke \& van den Ancker (2006). The upper limits to the size of the emitting region, are 0.25 arcsec corresponding to $39 \mathrm{AU}$ for HD 101412, 0.26 arcsec or $36 \mathrm{AU}$

\footnotetext{
1 http://wwW . eso.org/instruments/uves/

2 http://www . eso.org/projects/dfs/dfs-shared/web/ uves/uves-pipe-recipes.html
}

for HD $135344 \mathrm{~B}$, and 0.22 arcsec or 53 AU for HD 179218. These sizes represent the radii that contain $95 \%$ of the $6300 \AA$ [OI] line flux. The observations were completed at two different dates, to coincide with VTLI/MIDI observations of the same targets, in the mid-infrared ( $N$-band). Observations acquired using different spectroscopic-slit angles, were almost identical and were therefore average-combined. The data acquired at different nights however show temporal change and therefore are handled independently. They will be referred to as B1 (May 15-17), and B2 (June 16 and July 3, 4) in this paper.

We calculated the radial velocity of our targets using the difference between the measured line center and the theoretical line center, for 9 to 31 atmospheric absorption lines observed in the same spectrum. Errors have been determined by bootstrapping the data set, i.e. by estimating the variability of the radial velocity from the variability of this velocity between sub samples of the complete data-set. The size of the sub-samples used are $90 \%$ of the total sample and we have estimated the variability by calculating the mean of the sub-sample a 1000 times. The results are shown in Table 2. The [OI] $6300 \AA$ line is located between several telluric absorption lines. To remove the telluric absorption lines, we fit and fill the lines using a Gaussian, and average the telluric line region over a wavelength range 1.5 times the measured FWHM of the line to suppress telluric line residuals. These telluric absorption lines only overlap the [OI] spectral region on the red wing of HD 101412. The results are shown in Figs. 1-3. In these figures, we also show the location of the $6300 \AA[\mathrm{OI}]$ sky emission line. This line has been removed by calculating the sky emission from averaging above and below the echelle order, and subtracting this from the final spectrum. Because the sky emission is weak compared to the flux collected from the targets, the procedure has no significant effect on either the resulting spectrum or the signal to noise.

The spectra were corrected for their radial and barycentric velocity. We normalized and then converted the spectra, to velocity profiles about the central wavelength of the [OI] $6300 \AA$ line $(6300.304 \AA)$. The resulting spectra of HD 101412 and HD 179218, are shown in Figs. 4 and 6. The spectrum of HD 135344 B in Fig. 5 needs to undergo one more reduction step to correct for the underlying photospheric absorption lines, as discussed in Sect. 4.2.2.

The spectra of HD 101412 and HD 179218 both show a double peaked [OI] line-profile indicating the presence of a circumstellar disk, and temporal variance indicating disk inhomogeneity. HD 101412 shows a maximum signal at a level of $10 \%$ above the continuum, has wings that are $60 \mathrm{~km} \mathrm{~s}^{-1}$ wide and, next to the double peak, displays two extra "shoulders". The data also show temporal change, with a more pronounced double peak being evident in the B1 data in addition to more flux in the "shoulders". In Fig. 4, we deduce that, in the B2 data, the difference between the two lines on the red wing at $50 \mathrm{~km} \mathrm{~s}^{-1}$, is not caused by temporal variability because the spectrum drops below the mean continuum. It is not present in the [OI] emission line at $6363 \AA$, and must therefore represent real absorption. This line should be present at wavelengths that correspond to telluric absorption in the B1 data, and is therefore not detected in that spectrum. We compared the spectrum with that of HD 34364 , another B9.5V star, but failed again to identify the absorption line, ruling out the presence of photospheric absorption. We exclude the possibility telluric emission because the width of the line is $15 \mathrm{~km} \mathrm{~s}^{-1}$, larger than the telluric line width of $4 \mathrm{~km} \mathrm{~s}^{-1}$. We therefore conclude that this absorption line is probably interstellar, but are unable to identify its precise origin. During further 
Table 2. Astrophysical parameters of the programme stars.

\begin{tabular}{ccccccccc}
\hline \hline Name & Spectral Type & $\begin{array}{c}\log T_{\text {eff }} \\
\log [\mathrm{K}]\end{array}$ & $\begin{array}{c}\log L_{\text {bol }} \\
\log \left[L_{\odot}\right]\end{array}$ & $\begin{array}{c}L_{\text {IRE }} \\
{\left[L_{\star}\right]}\end{array}$ & $\begin{array}{c}M \\
{\left[M_{\odot}\right]}\end{array}$ & $\begin{array}{c}\text { Distance } \\
{[\mathrm{pc}]}\end{array}$ & $\begin{array}{c}v_{\text {rad }} \\
{\left[\mathrm{km} \mathrm{s}^{-1}\right]}\end{array}$ & $\begin{array}{c}v \sin i \\
{\left[\mathrm{~km} \mathrm{~s}^{-1}\right]}\end{array}$ \\
\hline HD 101412 & A0IIIe & 4.02 & 1.40 & 0.27 & $2.3 \pm 0.2$ & $160^{a}$ & $16.9 \pm 0.2$ & $8 \pm 1$ \\
HD 135344 B & F4Ve & 3.82 & 1.01 & 0.43 & $1.7 \pm 0.2$ & $140^{b}$ & $1.6 \pm 1.3$ & $75 \pm 5$ \\
HD 179218 & B9e & 4.02 & 1.88 & 0.28 & $2.7 \pm 0.3$ & $240^{c}$ & $15.4 \pm 2.3$ & $72 \pm 5$ \\
\hline
\end{tabular}

\begin{tabular}{|c|c|c|c|c|c|c|}
\hline Name & $\begin{array}{l}{[\mathrm{OI}] E W} \\
[\AA]]\end{array}$ & $\begin{array}{c}L([\mathrm{OI}]) \\
{\left[L_{\odot}\right]}\end{array}$ & $\begin{array}{c}{[\mathrm{OI}] \text { inner radius }} \\
{[\mathrm{AU}]}\end{array}$ & $\begin{array}{c}\text { [OI] outer radius } \\
{[\mathrm{AU}]}\end{array}$ & $\begin{array}{c}\text { Inclination } \\
{\left[{ }^{\circ}\right]}\end{array}$ & Group \\
\hline HD 101412 & $-0.13 \pm 0.01$ & $(9.5 \pm 0.5) \times 10^{-5}$ & 0.15 & 10 & 30 & II \\
\hline HD 135344 B & $-0.13 \pm 0.06$ & $(1.3 \pm 0.5) \times 10^{-4}$ & 0.1 & 90 & $45^{d}$ & I \\
\hline HD 179218 & $-0.053 \pm 0.005$ & $(3.4 \pm 0.3) \times 10^{-4}$ & 0.4 & 65 & $40^{e}$ & I \\
\hline
\end{tabular}

${ }^{a}$ Acke et al. (2005). ${ }^{b}$ van Boekel et al. (2005). ${ }^{c}$ van den Ancker et al. (1998). ${ }^{d}$ Doucet et al. (2006). ${ }^{e}$ Dent et al. (2005).

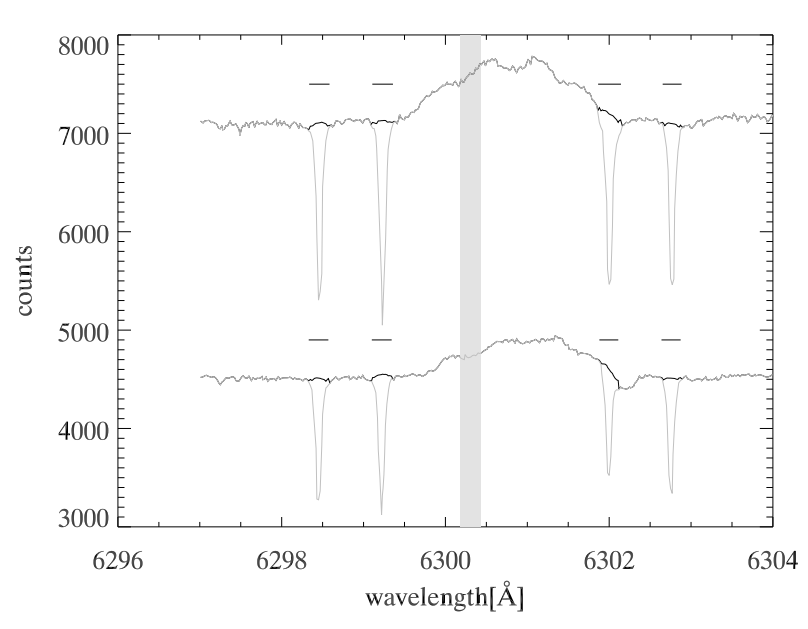

Fig. 1. The spectrum for HD 101412. The clipped region is shown in black for the B1 (upper line) and the B2 (lower line) spectra, and is also shown with horizontal bars above the clipped parts. The grey area denotes the location of the (subtracted) $6300 \AA[\mathrm{OI}]$ sky line. The emission is not symmetric around this line because these spectra are not yet corrected for the radial and barycentric velocity.

analysis, we replace the affected part of the spectrum between 35 and $65 \mathrm{~km} \mathrm{~s}^{-1}$, with the data between -65 to $-35 \mathrm{~km} \mathrm{~s}^{-1}$, of the same spectrum ${ }^{3}$. We indicate this replacement with a grey dotted line, while the original absorption is shown with a grey solid line in Fig. 4.

The [OI] emission line observed for HD 179218 rises a maximum of $5 \%$ above the continuum, is $50 \mathrm{~km} \mathrm{~s}^{-1}$ wide, and shows intrinsic temporal variation. The red wing of the line changes between both data sets, which is not caused by normalizing effects.

The [OI] emission in HD $135344 \mathrm{~B}$ has a maximum approximately $8 \%$ above the continuum and a single-peaked profile. Because of the overlapping photospheric absorption-lines, it is difficult to determine accurately the velocity broadening of the line, which we estimate to be between 60 and $100 \mathrm{~km} \mathrm{~s}^{-1}$. The [OI] line does not vary significantly with time, in contrast to that of HD 101412 and HD 179218. The line reaches a peak at $3.9 \mathrm{~km} \mathrm{~s}^{-1}(3 \sigma)$ away from the expected, rest-frame velocity, measured using photospheric lines.

\footnotetext{
${ }^{3}$ In order to compare how much this changes our final results, we show the results obtained with the two separate wings in Fig. 11 as well as the results obtained with the above mentioned changes in Fig. 13.
}

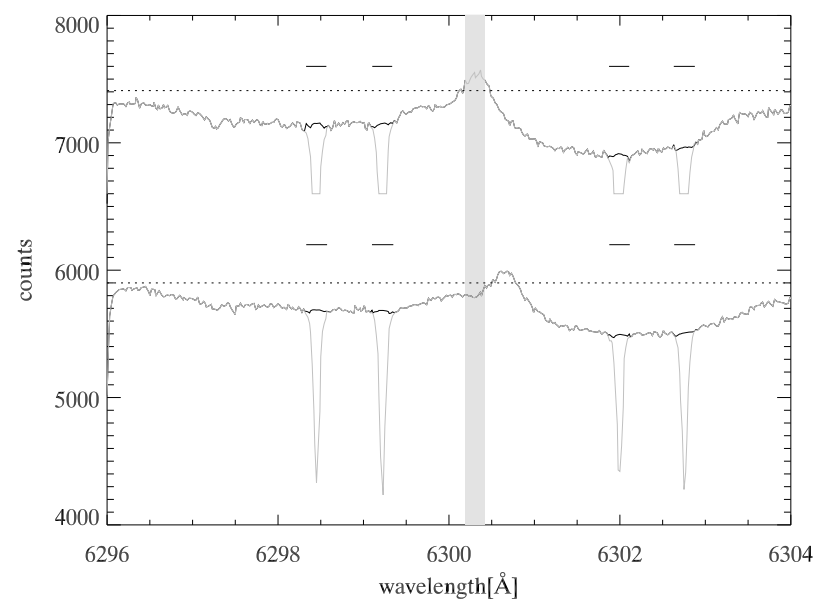

Fig. 2. Same as Fig. 1 for HD 135344 B. Note that the $6300 \AA$ [OI] line is mixed with broad atmospheric absorption lines. The over plotted horizontal dotted line indicates the continuum and the telluric absorption lines of the B1 data have been truncated for so it wont overlap with the B2 data.

\section{Stellar parameters}

The SEDs of HAEBE stars can be divided into two groups, based on the shape of the mid-IR $(20-100 \mu \mathrm{m})$ emission, which are referred to as groups I and II (Meeus et al. 2001). Group-I sources have a rising mid-IR flux-excess, indicative of a flared disk, while group-II sources display a more modest IR-excess, more typical of a geometrically-flat disk. This difference between the groups can be assessed more quantitatively using the $L_{\mathrm{NIR}} / L_{\mathrm{IR}}$ vs. IRAS [12]-[60] colour diagram (cf. Acke et al. 2004). In Figs. 7-9 we show the SED for each source. By using this classification and comparing SEDs, HD 179218 and HD 135344 B are classified to be group I sources. In the $L_{\mathrm{NIR}} / L_{\mathrm{IR}}$ vs. IRAS [12]-[60] diagram, HD 101412 is located close to the line separating the two groups. Although it is a group-II source, it may be considered a transitional object between both groups.

A Kurucz (1991) model-atmosphere, corresponding to the photospheric parameters of the central star, is fitted to the data to represent the photospheric contribution. From the model-fitting, the shape and excess emission from the disk become clear. The shortest wavelength for which the excess is discernible, is $0.9 \mu \mathrm{m}$ for HD 101412, $1.5 \mu \mathrm{m}$ for HD $135344 \mathrm{~B}$, and $1.0 \mu \mathrm{m}$ for HD 179218 . for the wavelengths larger than this point, the 


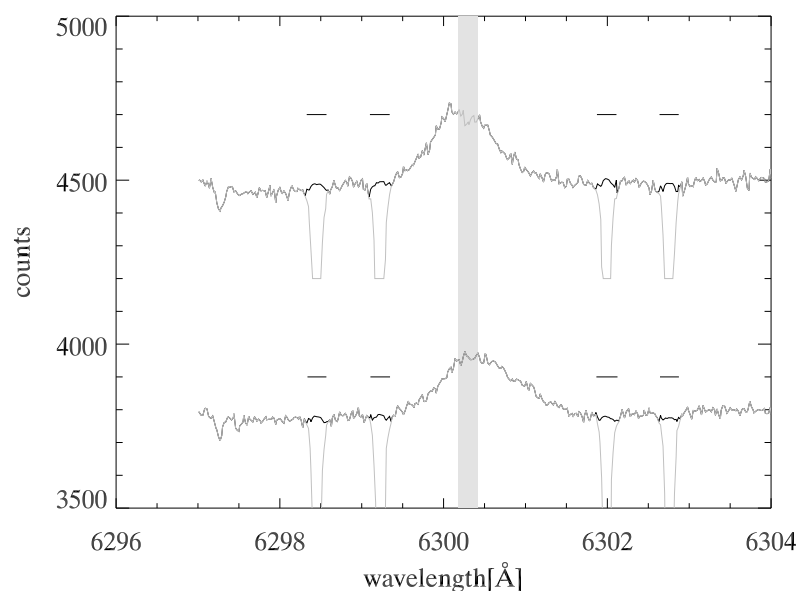

Fig. 3. Same as Fig. 1 for HD 179218 , the telluric absorption lines of the B1 data have been truncated for so they wont overlap with the B2 data.

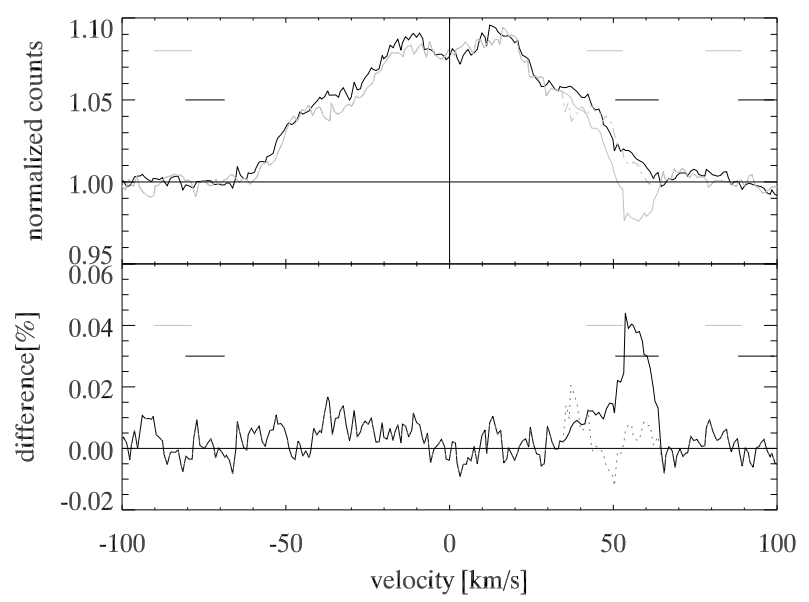

Fig. 4. In the top window the velocity spectrum of HD 101412 centered around the $[\mathrm{OI}]$ line, after correcting for barycentric and radial velocity and normalization, for B1 (black) and B2 (grey). The location of the clipped out telluric lines is shown by horizontal bars. The difference between both observations is shown in the bottom window. The interstellar absorption feature at $50 \mathrm{~km} \mathrm{~s}^{-1}$ discussed in Sect. 2 is clearly visible and replaced with the blue shifted part of the same spectrum as shown with the dotted line.

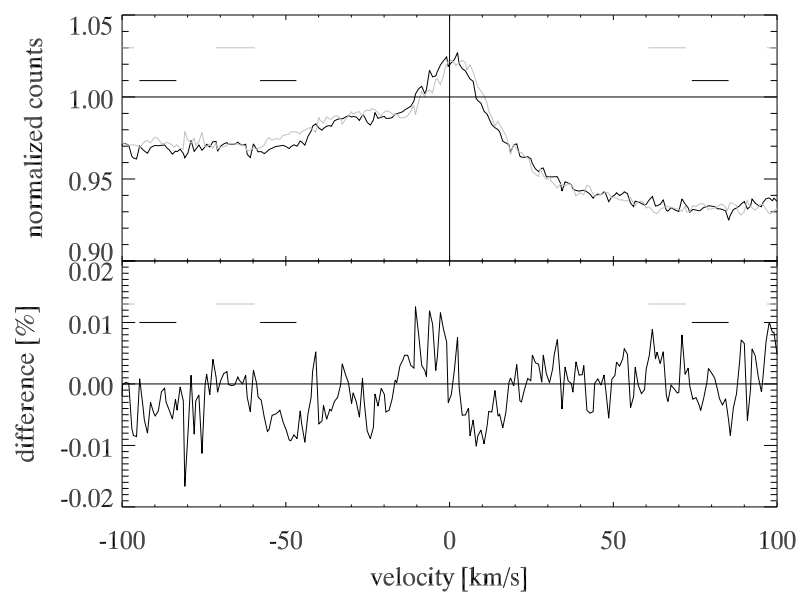

Fig. 5. Same as Fig. 4 for HD 135344 B.

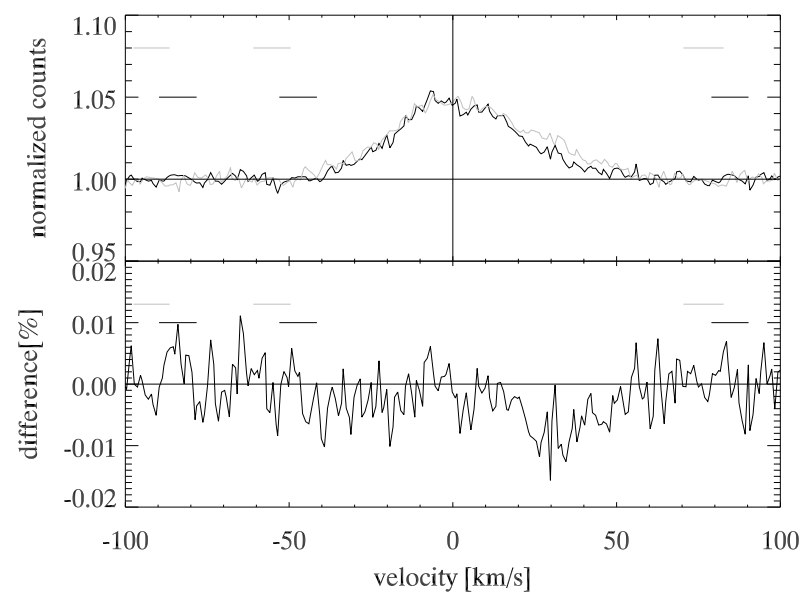

Fig. 6. Same as Fig. 4 for HD 179218.

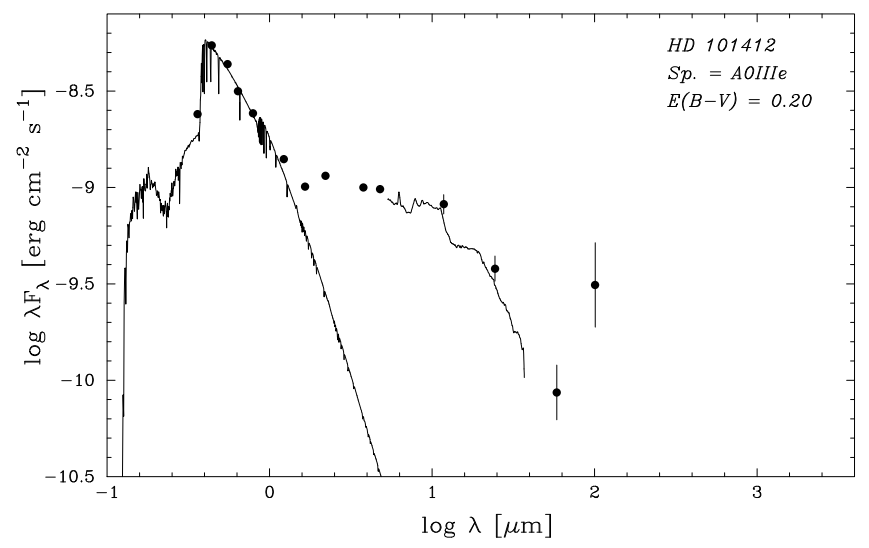

Fig. 7. The SED of HD 101412 compiled using literature photometry. The solid line is a reddened Kurucz stellar atmosphere model fitted to the photometry of the central star. Also shown are SPITZER-IRS data from Bouwman et al. (2007, in prep.).

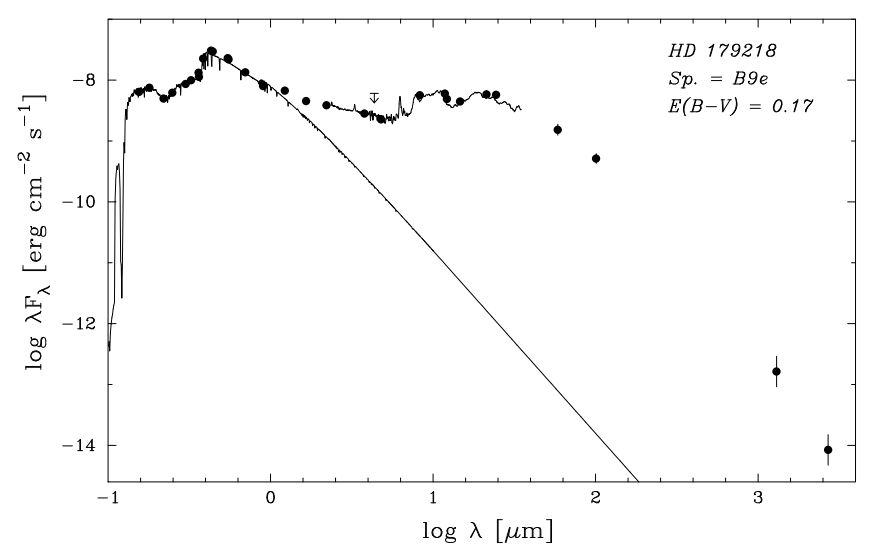

Fig. 8. Same as Fig. 7 for HD 179218 but the ISO-SWS spectrum is shown here (Acke \& van den Ancker 2004).

excess emission for HD 101412 shows a steady decline that "flares up" at $100 \mu \mathrm{m}$. This behavior is also displayed by other IRAS sources at long wavelengths, and is probably caused by the inclusion of interstellar material heated by, but not directly associated with, the central star in the rather large (1 arcmin) IRAS beam. The remaining two SEDs show a re-brightening at mid-IR 


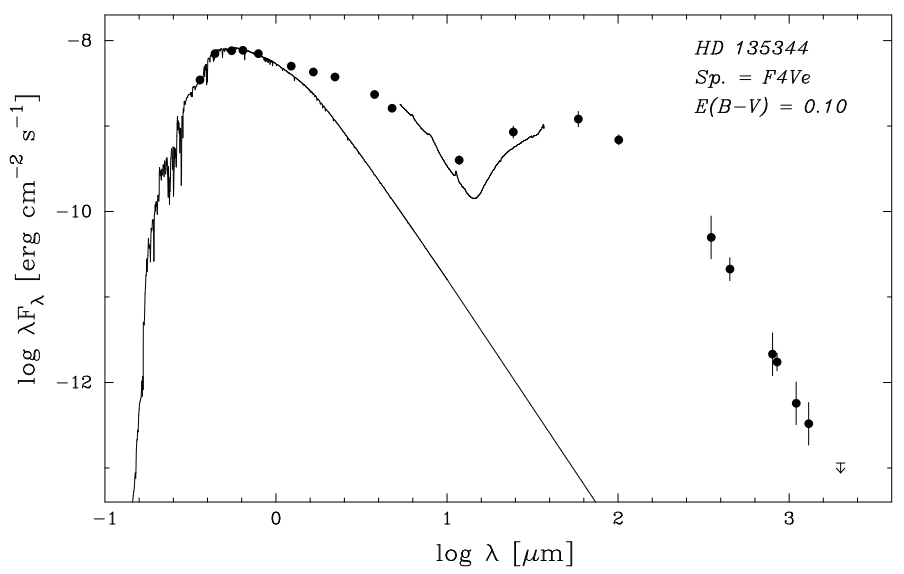

Fig. 9. Same as Fig. 7 for HD 135344 B.

wavelengths. The fractional luminosity of the dust compared to the stellar luminosity, $L_{\mathrm{IRE}} / L_{\star}$, is listed in Table 2.

To determine the projected rotational velocity, the $v \sin i$, we fitted a Doppler profile to mimic the broadening of the lines by stellar rotation to the rotational line profile of several atmospheric absorption lines.

\section{1. $H D 101412$}

HD 101412 has a spectral type between B9.5Ve (Houk \& Cowley 1975) and A0 III/IVe (Guimarães et al. 2006), and a distance of $160 \mathrm{pc}$. By integrating under the SED shown in Fig. 7 (using a reddened Kurucz model to interpolate between observed data points in the optical, a spline in the infrared and a grey body to extrapolate the last data points), and adopting the distance of $160 \mathrm{pc}$, we derive a bolometric luminosity of $25 L_{\odot}$ for HD 101412. The Spitzer-IRS spectrum of HD 101412 has an unusual shape, due to the presence of strong PAH emission bands on top of a plateau-like $10 \mu \mathrm{m}$ silicate feature over a weakly-rising continuum (Bouwman et al. 2007, in prep.). The same authors also note the presence of emission bands due to the presence of crystalline silicates at 19, 24, 28 and $34 \mu \mathrm{m}$. This presence of strong PAH emission is, similar to the presence of strong [OI] emission, unexpected for a group-II source (Acke \& van den Ancker 2004). This reinforces our suggestion that the disk of HD 101412 is in transition between flaring and being self-shadowed.

We estimate the mass of HD 101412, derived by comparing the position in the HR diagram to the theoretical pre-mainsequence tracks of Palla \& Stahler (1993), to be $2.3 \pm 0.2 M_{\odot}$. Guimarães et al. (2006) find a $v \sin i$ of $7 \pm 1 \mathrm{~km} \mathrm{~s}^{-1}$ by modeling high-resolution $(R=48000)$ spectra, suggesting that we are observing HD 101412 almost pole-on. This agrees well with the value derived from our data of $8 \pm 1 \mathrm{~km} \mathrm{~s}^{-1}$.

\section{2. $H D 135344 B$}

HD 135344 B is a wide visual binary with a separation of 19.6 arcsec located at a distance of $140 \mathrm{pc}$. In this paper, we will discuss component HD $135344 \mathrm{~B}$, which has spectral type F4Ve and an estimated mass of $1.7 \pm 0.2 M_{\odot}$. It has a bolometric luminosity of $10 L_{\odot}$, obtained by fitting the SED shown in Fig. 9. Bouwman et al. (2007, in prep.) note only very weak PAH features in the Spitzer-IRS spectrum of HD 135344 B. They also note that its SED is quite unusual, with an IR excess starting at $1.5 \mu \mathrm{m}$, but a deep bump at $13 \mu \mathrm{m}$. Doucet et al. (2006) have imaged HD $135344 \mathrm{~B}$ in the mid-infrared $(20.5 \mu \mathrm{m})$ and find a disk with an inclination of $45^{\circ}$ and a PA of $100 \pm 10^{\circ}$. Based on these spatially-resolved observations, they estimate that the disk extends up to $200 \mathrm{AU}$. Brown et al. (2007) classify the disk around HD 135344 B as a "cold disk", interpreting the lack of mid-IR emission in the SED as missing warm dust. From modeling the SED, they derive the disk to have an inner rim starting at $0.18 \mathrm{AU}$, a gap (i.e. missing warm dust) from 0.45 to $45 \mathrm{AU}$, and outside that, a gas rich disk.

A simple model-fit to the $\mathrm{CO}(J=3-2)$ double peaked line profile fitted by Dent et al. (2005), indicates an inner radius of the emitting gas $\leq 10 \mathrm{AU}$, an outer radius of $75 \pm 5 \mathrm{AU}$, an estimated circumstellar dust mass of $10^{-4} M_{\odot}$, and an inclination of $11 \pm$ $2^{\circ}$. The value of this inclination is notably different from the result of Doucet et al. (2006), and implies that the star rotates at breakup velocity. We will use the result of Doucet et al. (2006) in our model because it is obtained in a more direct way.

\section{3. $H D 179218$}

HD 179218 is a B9e star at a distance of $240 \mathrm{pc}$ and is the Herbig star with the 2nd largest known percentage of crystalline dust (van Boekel et al. 2005). It has a bolometric luminosity of $76 L_{\odot}$, obtained using the SED shown in Fig. 8. HD 179218 is the star with the greatest richness in terms of number of infrared spectral features in the overview of infrared spectra of Herbig Ae/Be stars by Bouwman et al. (2008, in prep.). Its Spitzer-IRS spectrum is dominated by strong emission features due to the presence of abundant crystalline silicates. Polycyclic Aromatic Hydrocarbons are also detected, in agreement with its classification as a group-I source (Acke \& van den Ancker 2004). We estimate the mass based on the position in the HR diagram to be $2.7 \pm 0.3 M_{\odot}$. Guimarães et al. (2006) find a $v \sin i$ of $72 \pm 3 \mathrm{~km} \mathrm{~s}^{-1}$, agreeing well with the value derived from our data of $72 \pm 5 \mathrm{~km} \mathrm{~s}^{-1}$.

Elia et al. (2004) fit the continuum spectrum of HD 179218 with a model including a polar cavity, suggesting a disk-like geometry in the innermost part of the envelope. Moreover, it is noteworthy that their model corresponds to a dust-absorption coefficient exponent $\beta=0.6$, which is typical of large dust grains and agrees with the results of Acke et al. (2004). This is in agreement with the idea that the grain size is expected to increase with time in circumstellar envelopes, as a result of the ongoing growth processes activated in high-density environments. Liu et al. (2007) have resolved warm dust using 10-micron nulling interferometric observations covering a diameter of $27 \pm 5 \mathrm{AU}$ around the star. Their observations suggest circular symmetry and thus a small inclination, but inclinations up to $45^{\circ}$ are still within the error bars. A simple model fit to the $\mathrm{CO}(J=3-2)$ line profile completed by Dent et al. (2005), implies an outer radius of $120 \pm 20 \mathrm{AU}$, an inclination of $40 \pm 10^{\circ}$, and an estimated circumstellar dust mass of $10^{-4} M_{\odot}$.

\section{Data analysis}

\subsection{Method}

In pioneering work on the mechanism responsible for [OI] emission in young stars, Hartigan et al. (1995) modeled the broad [OI] $6300 \AA$ line emission seen in T Tauri stars as being due to a combination of dense stellar jets and a disk wind or magnetic accretion columns. Acke et al. (2005) have developed a model that explains the narrow [OI] emission lines seen around many HAEBE stars. This simplified model assumes that the 


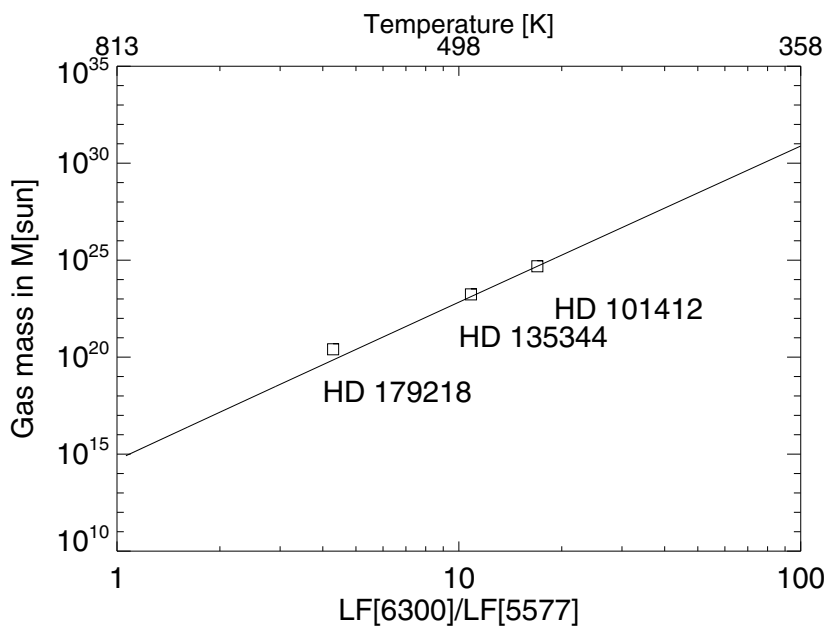

Fig. 10. Total mass of gas needed to explain the received [OI] $6300 \AA$ flux vs. the relative strength of the $5577 \AA$ vs. $6300 \AA$ line (lower $x$ axis) in case of thermal emission. The corresponding temperature that is needed to explain this ratio is plotted on the upper $x$-axis. Also shown are the data points for the 3 stars (squares) and the function connecting the ratio of Equivalent Widths to the total gas mass for HD 101412 (solid line). Because both lines are only resolved for HD 101412 we show lower limits for the other two targets. The disks around our three targets would need to be unrealistically massive to explain the observed $[\mathrm{OI}]$ emission as being due to thermal emission from the disk.

entire UV luminosity $(2-13.6 \mathrm{eV})$ of the central star (represented by a Kurucz model for the stellar photosphere) is used to photodissociate $\mathrm{OH}$ in the upper layers of the disk. The disk structure (i.e. densities and temperatures) is computed with a Chiang \& Goldreich (1997) type disk, with small olivine dust grains as the opacity source. The $\mathrm{OH}$ to $\mathrm{H}$ abundance is fixed a priori to be constant at each location in the disk. The absorption of UV photons by the dust in the disk, is taken into account in the same way as in the models of Störzer \& Hollenbach (2000) for photodissociation regions along the direction of the in-falling photons. The line profiles are measured by assuming Keplerian rotation and integration over the spatial coordinates. Details about the method we employ here can be found in Acke et al. (2005) and Acke \& van den Ancker (2006).

To verify the validity of this assumption for the three systems that we study we compare the Line Flux (LF) of the $5577 \AA[\mathrm{OI}]$ emission to that of the $6300 \AA$ [OI] emission. If this emission is caused by thermal emission originating from a disk with a temperature of $500 \mathrm{~K}$, the Boltzmann factor renders the ratio of these two to be very low (Fig. 10).

We follow Acke et al. (2005) and Acke \& van den Ancker (2006) in concluding that the observed [OI] emission is caused by illumination of the upper-disk layer by UV radiation.

We expect that the pattern and intensity of the [OI] emission is influenced by differences in disk geometry. The part of the disk that lies in the shadow of the puffed-up inner rim characteristic of group-II sources, will emit less compared to the parts that are directly illuminated by the central star. Therefore we predict that there is a drop in intensity at the location of the shadow cast by the inner rim in HD 101412. How extended this shadow is depends on when, and if, the disk rises out of the shadow again. We also expect the flaring disks to appear larger in [OI] emission, because they are illuminated by stellar UV flux at greater distance and with more intensity due to their shape than the self shadowing disks.

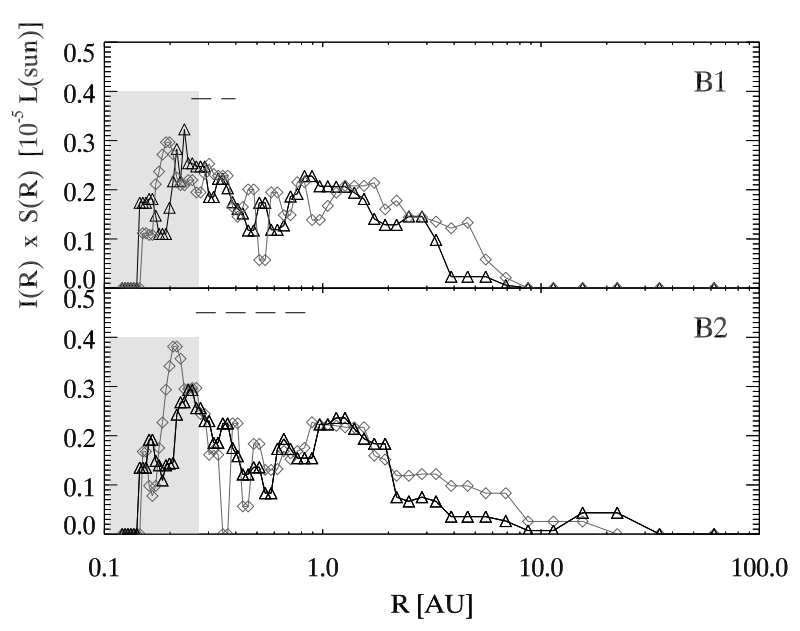

Fig. 11. The [OI] emission of the blue shifted (grey, diamonds) and red shifted (black, triangles) halves of the disk of HD 101412 . The two panels show the data taken at different times. The grey area is the suspected location of the inner rim. The horizontal dashed lines mark in the upper plot the location of the removed telluric absorption line (Fig. 1), and in the lower plot the part of the B2 spectrum that has been replaced as described in Sect. 2 and Fig. 4.

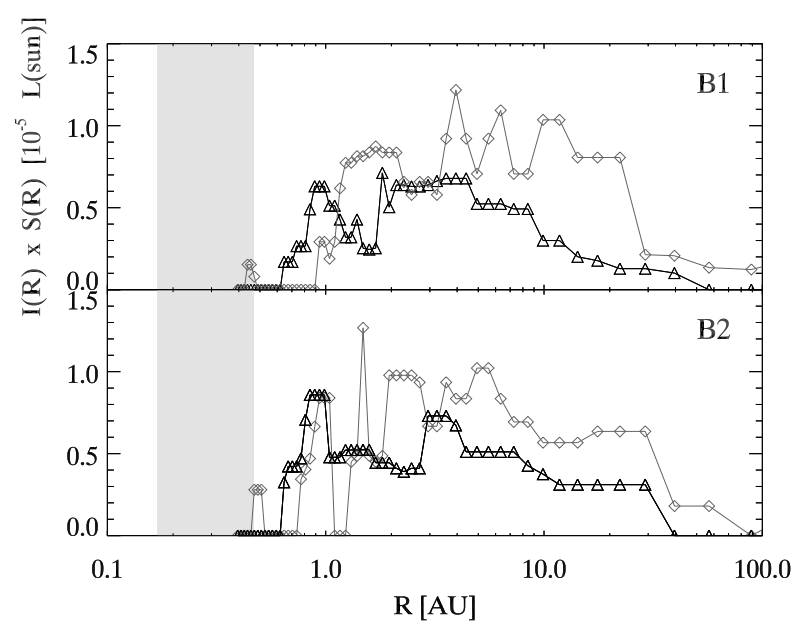

Fig. 12. Same as Fig. 11 for HD 179218.

We note that the radius scale of our results is dependent on the inclination of the disk used in the model. Taking a smaller inclination angle shifts the emission region closer to the star. If we compare the onset of the [OI] emission with the location of the dust-sublimation radius, we see that these agree for the inclinations used for HD $135344 \mathrm{~B}$ and HD 179218 . We use this to guess an inclination for HD 101412. Values between $24^{\circ}$ and $41^{\circ}$ allow the inner rim to be coincident with the dust sublimation radius. In the remainder of this paper, we will use a value of $30^{\circ}$. This low inclination is in agreement with both the low $v \sin i$, and the double-peaked line-profile observed in [OI].

\subsection{Results}

\subsubsection{HD 101412 and HD 179218}

By comparing the blue- and red-shifted Doppler profiles, it is possible to distinguish between the parts of the disk that rotate towards or away from us, and thus track possible 


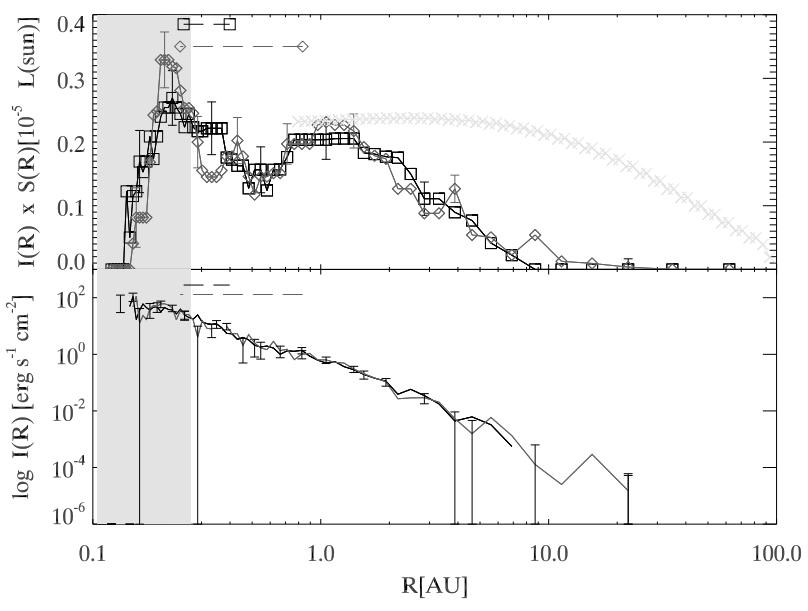

Fig. 13. The intensity versus radius graph of HD 101412 showing the distribution of $[\mathrm{OI}]$ emission as a function of distance from the central star. The two lines represent data taken on different dates; B1 is plotted in black with squares and B2 in grey with diamonds. The grey area is the suspected location of the inner rim and the light grey crossed line represents a simple model for [OI] emission from a flared disk in Keplerian rotation. In the upper plot we show the intensity normalized to the disk surface in a semi-log plot, as to better compare the inner and outer parts of the disk. A drop in intensity can clearly be seen at $0.6 \mathrm{AU}$ from the central star. In the lower plot we show the intensity derived from our model on a $\log -\log$ scale. The grey horizontal dashed lines marks the part of the red shifted part of the B2 spectrum that has been replaced as described in Sect. 2 and the black horizontal dashed lines marks the part of the red shifted part of the B1 spectrum of the removed telluric absorption line.

inhomogeneities. We have done this for HD 101412 and HD 179218 and show the results in Figs. 11 and 12 plotted together with the dust-sublimation radius for temperatures between 1200 and $2000 \mathrm{~K}$ in grey. The blue- and red-shifted wings of HD 101412 look approximately similar in the upper (B1) and bottom (B2) graph, apart from some excess flux at larger distances in the blue-shifted part of the disk. HD 179218 looks mildly asymmetric, showing for the red-shifted part emission that starts at a closer distance to the star and lacking intensity at larger distances compared to the blue-shifted part. The blue side also appears to harbor more flux and is detectable up to larger distances. The results discussed below for HD 101412 and HD 179218 are obtained by averaging the red- and blue-shifted profiles of both stars.

The distribution of emitting atomic oxygen gas as a function of radius for HD 101412, is plotted in Fig. 13, and for HD 179218 in Fig. 14. We plot the log - log dependency of the received flux as well as a semi-log-scale plot where we have normalized the intensity with the surface of the ring at distance $R: S(R)$, where $S(R)=\pi *\left(R_{\text {out }}^{2}-R_{\text {in }}^{2}\right)$ with $R_{\text {out }}$ and $R_{\text {in }}$ the outer and inner radius of the ring at distance $R$. In this sense, $I(R) x S(R)$ is the total luminosity in a ring at distance $R$, and the fluxes of the inner and outer part of the disk can be directly compared this way. The error bars drawn in these graphs are solely a function of the signal to noise of the data. The [OI] emission of HD 101412 starts within the expected location of the inner rim at $0.15 \mathrm{AU}$, and extends to $10 \mathrm{AU}$ with a clear drop of almost $50 \%$ in the $I(R)$ vs. $S(R)$ curve about 0.5 AU. Since HD 101412 is a groupII source, characterized by a puffed-up inner rim, and because the $[\mathrm{OI}]$-emission mechanism is directly proportional to (stellar) UV flux, the drop in intensity can be caused by the shadowing

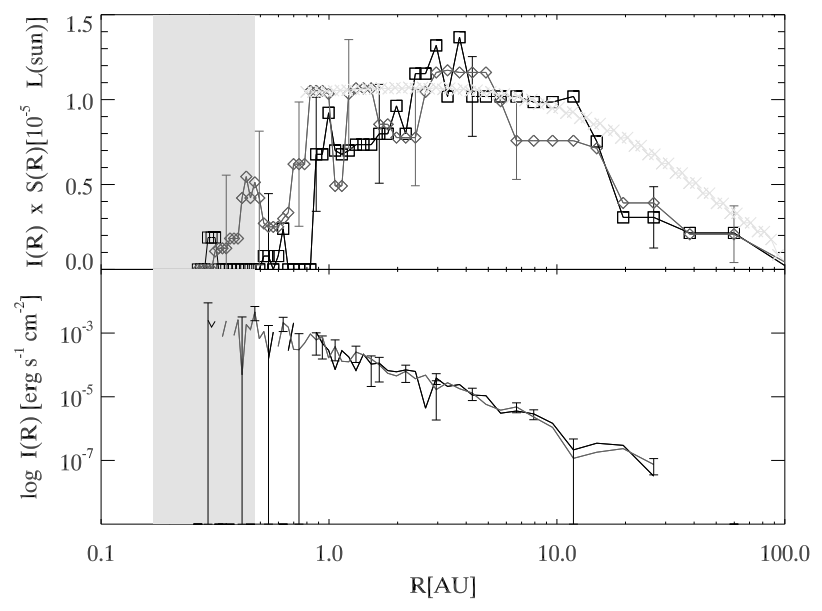

Fig. 14. Same as Fig. 13 for HD 179218 . The spikes at small radii and at $4 \mathrm{AU}$ are an artifact of our data reduction and not significant.

effect of the inner rim. Once the disk emerges out of the shadow (around $1 \mathrm{AU}$ ), the disk is again directly illuminated by the central star and continues to receive sufficient flux up to $10 \mathrm{AU}$ to be detectable. If we compare the [OI] emission of HD 101412 with that expected from a flared disk in Keplerian rotation, shown with light grey crosses in Fig. 13, one sees that for a flared disk, the emission should have been detected up to much larger radii. We interpret these discrepancies with the flared disk model that both can be explained by a self-shadowed disk; the local drop in intensity at $0.6 \mathrm{AU}$ and the disappearance of emission at $10 \mathrm{AU}$, as direct evidence that in the disk surrounding HD 101412, the inner rim indeed shadows part of the outer disk - as expected for a group II source.

The intensity vs. radius distribution for HD 179218, a group I source, looks different. It starts at $0.3 \mathrm{AU}$ from the central star, extends ${ }^{4}$ up to $65 \mathrm{AU}$ and has no clearly discernible local drop in intensity, as seen in HD 101412. This disk has a flaring geometry, meaning that it has - depending on the disk models used - no or negligible shadowing by the inner rim. The flaring also allows the disk to intercept more stellar flux at larger distance, causing the $[\mathrm{OI}]$ emission up to a larger distance from the central star. The onset of emission for the B2 data of HD 179218 is located beyond the dust sublimation radius. This result is reached by assuming the inclination found by Dent et al. (2005) of $40 \pm 10^{\circ}$. When the lower limit of the inclination of $30^{\circ}$ is assumed, the onset of emission starts at the dust sublimation radius. Both the shape and the inner plus outer radii of the $[\mathrm{OI}]$ emission are in reasonable agreement with those expected from a flareddisk model. The discrepancy between the observed and modeled emission at large radii is also seen in the two group I sources investigated by Acke \& van den Ancker (2006). This may be due to the model assumption that the $\mathrm{OH}$ abundance is homogeneous throughout the disk.

\footnotetext{
4 This number given here is larger than the in Sect. 2 inferred upper limit for the disk size. This is caused by the fact that the disk emission is concentrated towards the center and drops of exponentially as can be seen e.g. in the log-log part of Fig. 13. The the emission originating from $2 \mathrm{AU}$ of the central star already has dropped in intensity by a factor of 100 compared to the maximum.
} 


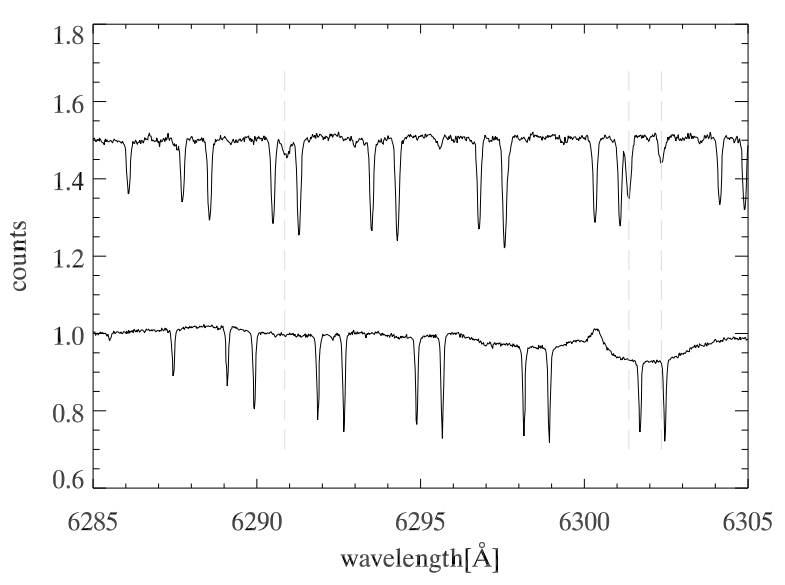

Fig. 15. The spectra of HD 135344 B (bottom) and HIP53070 (top), another F4V star but with low $v \sin i$. This plot shows that there are two broadened absorption lines marked with vertical lines mixing with the [OI] emission line of HD $135344 \mathrm{~B}$. All the other absorption lines are caused by telluric absorption.

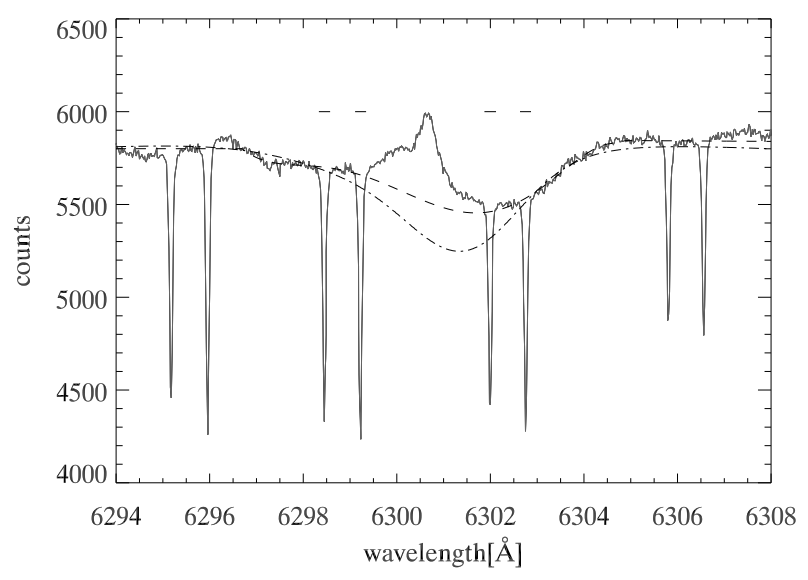

Fig. 16. The Chebyshev (dashed line) and the double Voigt (dot-dashed line) model used to model the photospheric absorption in HD 135344 B. The telluric absorption lines at the location of the four horizontal lines have been clipped out.

\subsubsection{HD $135344 \mathrm{~B}$}

The spectrum of HD 135344 B is contaminated by broad atmospheric absorption-lines. Because of this and because of the fact that the $[\mathrm{OI}]$ feature is overlapped by two of these lines as shown in Fig. 15, we undertook one extra step to reduce these data and therefore discuss this source separately. We correct the absorption by these two lines with two models: (1) a double Voigt model, because a Voigt profile closely mimics the expected profile of a stellar atmospheric line and (2) a polynomial model (a good fit was found using a 6th order Chebyshev-polynomial), both shown in Fig. 16. A problem when fitting the Voigt profile was that we were unable to fit the absorption using the location of the lines found in HIP 53070, a reference star with the same spectral type as HD 135344 B (Fig. 15). Using these values results in over (under) fitting the right (left) wing of the absorption. For the further modeling of HD 135344 B, we use these two models and their average, as shown in Fig. 17. This figure also shows that the blue- and red-shifted sides are asymmetric. This can be caused by an asymmetry in the emitting region, or by an

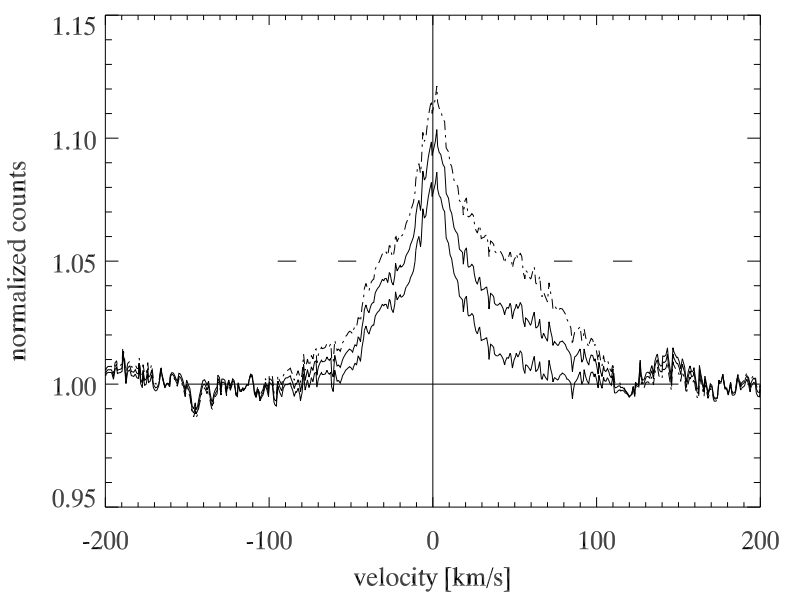

Fig. 17. The velocity corrected, normalized and converted to velocity around the [OI] line spectra corrected for the photospheric absorption using the 3 models of HD 135344 B. From top to bottom: the double Voigt model, the average model and the Chebyshev model. The horizontal bars mark the location of the clipped out telluric absorption lines. Only the spectra of B1 are shown.

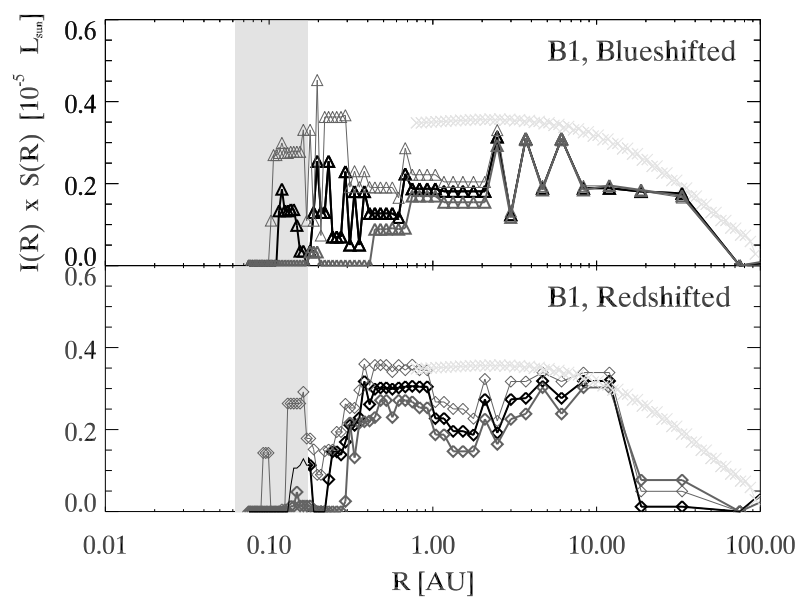

Fig. 18. The intensity versus radius graph for the blue (upper graph, triangles) - and red (lower graph, diamonds) shifted sides of HD 135344 B showing the distribution of $[\mathrm{OI}]$ emission as a function of distance from the central star. The three lines represent the B1 measurements for the Voigt model (upper grey line), the Chebyshev model (lower grey line) and the average of the two (thick black line). the light grey smooth line represents a simple model for the [OI] emission from a disk in Keplerian rotation.

imprecise correction for the photospheric absorption. Close inspection of Fig. 15 suggests that the blue side of the line, lies on a monotonically-increasing slope, while the red side of the line may overlap with the minimum of the mixed absorption line. Because of this, the blue- and red-shifted sides are considered separately in Figs. 18 and 19. There is a difference between the red and blue wing in the first AU, as well as between different models and data acquired at different times. The blue-shifted profile shows a steep initial rise followed by a large drop resembling the profile of HD 101412, whereas the red side rises more slowly, and displays a smaller drop in intensity at a larger radius. There is also a large difference between the two models (and thus also their average) used in the first couple of AU. For the Chebyshev model, the emission consistently starts at larger 


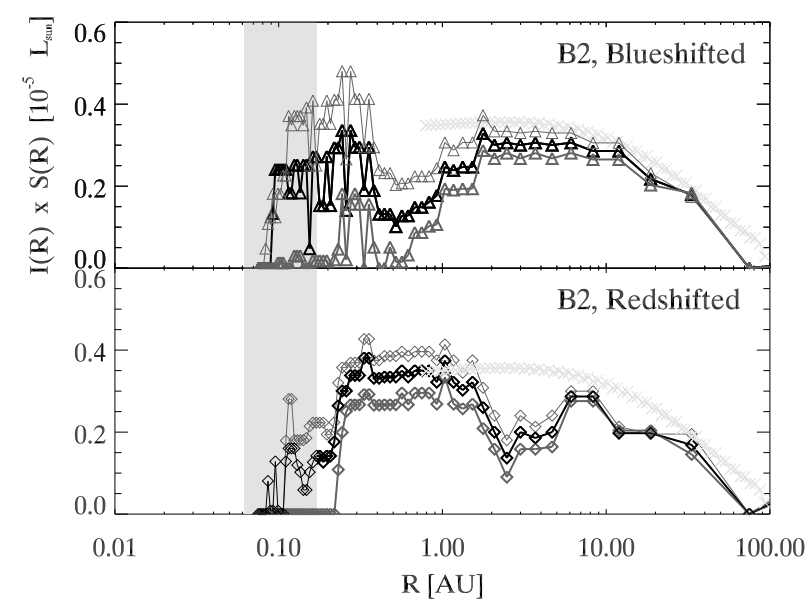

Fig. 19. Same as Fig. 18 for the B2 measurements.

radii than that of the Voigt model. In all cases however, the emission extends out to greater distance, and to higher luminosities than the other two sources, and vanishes around 90 AU. This value is comparable to the outer radius of $\mathrm{CO}$ gas of $75 \pm 5 \mathrm{AU}$ from Dent et al. (2005).

Considering (1) that it is not possible to properly correct for photospheric absorption, (2) the fact that HD $135344 \mathrm{~B}$ has an SED that is uncharacteristic of either a flaring or a self-shadowing disk because of the high (near) infrared excess between 1 and $4 \mu \mathrm{m}$ and (3) the fact that the peak of the [OI] emission velocity distribution does not coincide with the stellar photosphere, it is likely that the observed [OI] emission could be influenced by other components than solely a disk, such as an outflow with thermally excited oxygen. We therefore limit our interpretation of our [OI] data on this star to a phenomenological description of the spectra.

\section{Discussion and conclusions}

We have detected non-thermal [OI] emission in all three targets, one of which shows a double-peaked emission profile, and another one showing a tentative double peak, but certainly flattened emission profile, indicating emission from a circumstellar disk. We see in the [OI] emission of one of these sources, HD 101412, evidence for the existence of a puffed-up inner rim followed by a shadow, supporting the disk model of Dullemond et al. (2001). Although this model can explain most of our observations, there is one unexpected feature; the re-brightening (the 2nd bump) of the disk of HD 101412, shortly after the shadow cast by the inner rim. We expect the disk to remain in the shadow for a much longer distance, and possibly never re-emerge, preventing the disk becoming brighter. We offer a possible explanation for this behavior by speculating that the dust could have a lower scale height than the gas, allowing for a layer of gas above the dust disk. This layer may well (partially) rise above the shadow of the inner rim causing the behavior seen in Fig. 13. The [OI] emission of HD 179218 is in good agreement with emission from the surface of a flaring circumstellar disk inclined at a 30 degree angle. Finally, we encounter several difficulties in explaining the [OI] emission of HD 135344 B by a disk, and therefore suggest that more components, besides the disk, are responsible for the emission.

In future papers, we plan to improve our modeling. We plan to present MIDI observations of these stars showing warm dust, which will enable us to compare the coupling between gas and dust. We will model all observations using model SEDs. We propose to further constrain as many stellar parameters of our targets, as possible.

Acknowledgements. The authors of this paper wish to thank J. Liske for his assistance with modeling the atmospheric absorption lines. We also want to thank the referee for his helpful suggestions that helped to improve the presentation of the results in this paper.

\section{References}

Acke, B., \& van den Ancker, M. E. 2004, A\&A, 426, 151

Acke, B., \& van den Ancker, M. E. 2006, A\&A, 449, 267

Acke, B., van den Ancker, M. E., Dullemond, C. P., van Boekel, R., \& Waters, L. B. F. M. 2004, A\&A, 422, 621

Acke, B., van den Ancker, M. E., \& Dullemond, C. P. 2005, A\&A, 436, 209

Bik, A., \& Thi, W. F. 2004, A\&A, 427, L13

Bitner, M. A., Richter, M. J., Lacy, J. H., et al. 2007, ApJ, 661, L69

Blake, G. A., \& Boogert, A. C. A. 2004, ApJ, 606, L73

Blum, R. D., Barbosa, C. L., Damineli, A., Conti, P. S., \& Ridgway, S. 2004, ApJ, 617, 1167

Brown, J. M., Blake, G. A., Dullemond, C. P., et al. 2007, ApJ, 664, L107

Carmona, A., van den Ancker, M. E., Henning, T., et al. 2008, A\&A, 477, 839

Carr, J. S., Tokunaga, A. T., \& Najita, J. 2004, ApJ, 603, 213

Chiang, E. I., \& Goldreich, P. 1997, ApJ, 490, 368

Dekker, H., D’Odorico, S., Kaufer, A., Delabre, B., \& Kotzlowski, H. 2000, in Presented at the Society of Photo-Optical Instrumentation Engineers (SPIE) Conference, Optical and IR Telescope Instrumentation and Detectors, ed. M. Iye, \& A. F. Moorwood, Proc. SPIE, 4008, 534

Dent, W. R. F., Greaves, J. S., \& Coulson, I. M. 2005, MNRAS, 359, 663

Doucet, C., Pantin, E., Lagage, P. O., \& Dullemond, C. P. 2006, A\&A, 460, 117

Dullemond, C. P., Dominik, C., \& Natta, A. 2001, ApJ, 560, 957

Elia, D., Strafella, F., Campeggio, L., et al. 2004, ApJ, 601, 1000

Guimarães, M. M., Alencar, S. H. P., Corradi, W. J. B., \& Vieira, S. L. A. 2006,

A\&A, 457, 581

Hartigan, P., Edwards, S., \& Ghandour, L. 1995, ApJ, 452, 736

Hillenbrand, L. A., Strom, S. E., Vrba, F. J., \& Keene, J. 1992, ApJ, 397, 613

Hirth, G. A., Mundt, R., \& Solf, J. 1994, A\&A, 285, 929

Hirth, G. A., Mundt, R., \& Solf, J. 1997, A\&AS, 126, 437

Houk, N., \& Cowley, A. P. 1975, Michigan Catalogue of two-dimensional spectral types for the HD star, Ann Arbor: University of Michigan, Departement of Astronomy

Kurucz, R. L. 1991, in Precision Photometry: Astrophysics of the Galaxy, ed. A. G. D. Philip, A. R. Upgren, \& K. A. Janes, 27

Liu, W. M., Hinz, P. M., Meyer, M. R., et al. 2007, ApJ, 658, 1164

Mannings, V., \& Sargent, A. I. 1997, ApJ, 490, 792

Martin-Zaïdi, C., Lagage, P.-O., Pantin, E., \& Habart, E. 2007, ApJ, 666, L117

Meeus, G., Waters, L. B. F. M., Bouwman, J., et al. 2001, A\&A, 365, 476

Najita, J., Carr, J. S., \& Mathieu, R. D. 2003, ApJ, 589, 931

Natta, A., Prusti, T., Neri, R., et al. 2001, A\&A, 371, 186

Palla, F., \& Stahler, S. W. 1993, ApJ, 418, 414

Störzer, H., \& Hollenbach, D. 2000, ApJ, 539, 751

van Boekel, R., Min, M., Waters, L. B. F. M., et al. 2005, A\&A, 437, 189

van den Ancker, M. E., de Winter, D., \& Tjin A Djie, H. R. E. 1998, A\&A, 330, 145 